\title{
Multilinguales
}

\section{Le monologue racinien ou l'impossible dialogue}

Racinien Monologue or the Impossible Dialogue

\section{Christelle Stephan Hayek}

\section{(2) OpenEdition}

\section{Journals}

Édition électronique

URL : http://journals.openedition.org/multilinguales/1187

DOI : 10.4000/multilinguales. 1187

ISSN : 2335-1853

\section{Éditeur}

Université Abderrahmane Mira - Bejaia

\section{Édition imprimée}

Date de publication : 1 décembre 2014

Pagination : $30-49$

ISSN : 2335-1535

\section{Référence électronique}

Christelle Stephan Hayek, « Le monologue racinien ou l'impossible dialogue », Multilinguales [En ligne], 4 | 2014, mis en ligne le 01 décembre 2014, consulté le 17 septembre 2019. URL : http:// journals.openedition.org/multilinguales/1187; DOI : 10.4000/multilinguales.1187

Ce document a été généré automatiquement le 17 septembre 2019.

\section{(c) (i) ()}

Multilinguales est mise à disposition selon les termes de la Licence Creative Commons Attribution Pas d'Utilisation Commerciale - Pas de Modification 4.0 International 


\title{
Le monologue racinien ou l'impossible dialogue
}

Racinien Monologue or the Impossible Dialogue

\author{
Christelle Stephan Hayek
}

1 Dans la majorité des pièces raciniennes, nous retrouvons la forme - pourtant, déjà « démodée » à l'époque - du monologue. Obéissant à des règles plus ou moins strictes d'écriture et de dramaturgie, le monologue est, au théâtre, une scène où un personnage, seul sur scène, parle à lui-même.

2 Il est sans doute intéressant de voir dans quelle mesure on peut parler seul, sachant que la parole sert, par définition, à communiquer et que la communication suppose un locuteur et un destinataire.

\section{La communication théatrale}

Comme Piaget l'écrit si bien, "[le] parleur solitaire s'en prend parfois à des interlocuteurs fictifs» $(1997: 13)$, et Larthomas de corriger «[peut-être] faudrait-il remplacer parfois par toujours. Parler, c'est donc parler à autrui » (1980: 255). En effet, du moment qu'elle est proférée, la parole suppose - attend - qu'on l'écoute. Ne pouvant sans doute se résoudre à parler pour personne, le personnage se figure des destinataires possibles réels ou imaginaires - mais invariablement absents, qui tendraient pourtant l'oreille.

Le schéma de communication s'en trouve alors dévié de ses caractéristiques initiales et remodelé selon les besoins de cette forme de communication spécifique que l'on retrouve au théâtre. C'est ainsi que nous assistons à un véritable jeu des pronoms personnels qui s'efforcent de désigner les divers pôles de la communication, démêlant un « je » caméléon d'un « tu » volatil.

5 Toute situation de communication se fond, théoriquement, et dans un premier temps, dans le moule proposé par Roman Jakobson en 1960, à savoir le schéma suivant (Jakobson, $1960: 214$ ) : 


\begin{tabular}{|l|l|l|l|l|}
\hline & & Contexte & & \\
\hline Destinateur & & Message & & Destinataire \\
\hline & & Contact & & \\
\hline & & Code & & \\
\hline
\end{tabular}

6 Dans le cas du théâtre, le schéma est plus complexe, et ses différents pôles se dédoublent et se diversifient. C'est ainsi que

la représentation théâtrale est un ensemble (ou un système) de signes de nature diverse, relevant sinon totalement, du moins partiellement, d'un procès de communication, puisqu'elle comporte une série complexe d'émetteurs (en liaison étroite les uns avec les autres), une série de messages (en liaison étroite et complexe les uns avec les autres, selon des codes extrêmement précis), un récepteur multiple, mais situé en même lieu. (UBERSFELD, I, $1996: 20$ )

Il convient donc d'abord de cerner les deux actants de la communication théâtrale, à savoir la « série d'émetteurs » et le « récepteur multiple».

Dans son Dictionnaire du théâtre (1987), Patrice Pavis présente la problématique de la « communication théâtrale » caractérisée par plusieurs axes possibles : personnage / spectateur, personnage / personnage, comédien / spectateur, comédien / comédien, spectateur / spectateur (pp. 87-90).

Les trois derniers axes (spectateur, comédien / comédien, spectateur / spectateur) ne nous intéressent pas parce qu'ils se situent hors du texte théâtral à proprement parler.

Par ailleurs, selon Ubersfeld, dans ce type de communication, il y aurait deux émetteurs: l'émetteur 1, qui est l'auteur, et l'émetteur 2, qui est l'ensemble des praticiens ou des acteurs (Ubersfeld, II, $1996: 1$ ).

Mais, il faudrait sans doute ajouter à ces deux émetteurs successifs, un troisième, qui serait le personnage dont le rôle est joué par le praticien. Pourtant, son statut en tant qu'émetteur pourrait être contesté vu qu'il est défini comme " une unité lexicalisée ", un ensemble formé d'un « sémème » et d'un « lexème » (Ubersfeld, II, 1996: 150), et qu'il "n'a d'autre existence que celle de la fiction, [puisque] c'est un "être de papier " ». En d'autres termes, "[Il] ne prend forme que par sa présence sur scène, par les paroles qu'il prononce ou que l'on prononce sur lui » (David, 1995 : 142).

L'émetteur n'est donc autre que l'acteur, car «le seul individu concret qui figure sur la scène, c'est le comédien » (Ubersfeld, II, 1996 : 150).

Pourtant, quand nous lisons une pièce de théâtre - et certaines pièces, comme Lorenzaccio d'Alfred de Musset (1834), étaient à l'origine destinées à être lues - c'est le personnage qui prend en charge le message qu'il transmet. Le comédien est absent.

Nous pouvons ainsi avancer que la communication, dans le cadre d'une pièce de théâtre, se situe à trois niveaux :

1. l'auteur, le « je-scripteur [qui] parle un discours éclaté en plusieurs voix » et qui s'adresse « à vous spectateurs » et/ou lecteurs (Ubersfeld, III, $1996: 53$ ) ;

2. l'acteur qui prend en charge la transmission du discours de l'énonciateur premier pour le faire parvenir au spectateur ;

3. le personnage qui communique avec un autre personnage, dans le cadre de la fiction. 


\section{Le monologue théatral}

7 Nous considérerons ici, le troisième niveau de la communication ${ }^{1}$, à savoir la situation du personnage de théâtre qui se retrouve, le temps de son monologue, seul sur scène, sans destinataire physique, à son niveau de la communication. D'ailleurs «les lois $d u$ discours concernent les seuls personnages. » (Maingueneau, 1990 :145).

Dans ce cas-là, on pourrait éventuellement considérer le spectateur comme le véritable récepteur du message puisqu'il le perçoit et le comprend. Mais à la différence de la tragédie grecque où «le message tragique n'est transparent [...] que pour le spectateur [...] [qui] est appelé à déchiffrer fable et partition à travers la représentation [...] [puisque] lui seul peut [les] interpréter» (Girard, Ouellet, Rigault, $1986: 168$ ), chez Racine, par contre, «le personnage assume à la fois son rôle, celui du chœur et celui du spectateur » (Bakhtine, 1984 : 336), à savoir la fonction du récepteur et du décodeur du message. C'est ainsi que «le "public" est moins un partenaire qu'un voyeur» (Bakhtine, 1984 : 336). Le spectateur ne " participe » donc pas à la communication théâtrale.

Dans un monologue, le locuteur est toujours le personnage présent sur scène, mais ce qui est remarquable, c'est que ce monologuiste parle seul. Or, par définition, le verbe « parler » suppose un destinataire, une oreille attentive et conseillère et selon Bakhtine (1984:336) :

L'énoncé a toujours un destinataire (aux caractéristiques variables, qui peut être plus ou moins proche, concret, perçu avec une conscience plus ou moins grande) dont l'auteur de la production verbale attend et présume une compréhension responsive.

Donc, «Racine, pour conférer au monologue un maximum d'intensité dramatique, lui donne [...] la forme d'un dialogue fictif» (Hubert, 2003: 94). De plus, le rôle du destinataire est assuré, par définition, du fait qu'un monologue est «théâtre, [par] un allocutaire présent et muet, le spectateur » (Ubersfeld, III, 1996 : 21).

Donc même si le personnage est seul sur la scène, l'autre, qui écoute, est toujours là, sous une forme ou une autre, puisqu'en cas d'absence d'un des pôles du schéma, la situation de communication est faussée.

Le seul élément manquant de la situation de communication qu'est le monologue, par le fait même de la parole dite, est le destinataire qui est donc recréé, par le pouvoir de l'imagination du locuteur. À défaut d'une oreille attentive, le destinataire se voit prendre les identités les plus diverses: «Ainsi, le personnage, pour rompre sa solitude, interpellera un objet [...] ou un dieu sous la forme d'une prière ou d'imprécations " (Girard, Ouellet, Rigault, $1986: 39$ ).

Souvent, le personnage, dans sa solitude, se dédouble, devenant en même temps l'émetteur et le récepteur. Or, il ne peut remplir ce double rôle qu'en alternant les pronoms personnels qui le désignent.

En effet, le «je », par définition, désigne l'émetteur, celui qui produit le message, et " chaque je a sa référence propre ; [...] [il] est l'individu qui énonce la présente instance de discours contenant l'instance linguistique je » (Benveniste, $1974: 251)$. Il ne peut, en aucun cas, remplacer le destinataire à qui appartient exclusivement le pronom «tu ». Celui-ci désigne "l'individu allocuté dans la présente instance de discours contenant l'instance linguistique tu » (Benveniste, 1974 : 252).

Le personnage se voit ainsi contraint à être lui-même et un autre à la fois; celui qui se plaint et celui qui console, celui qui ordonne et celui qui exécute les ordres. D’ailleurs, selon Benveniste, 
[le] monologue est un dialogue intériorisé, formulé en langage intérieur, entre un moi locuteur et un moi écouteur [...] [qui] s'énonce [tantôt] comme "première personne ", [...] tantôt [...] comme " deuxième personne » (Benveniste, 1974 : 251).

Or, ce dédoublement n'est possible que si les deux « moi » en présence sont distincts.

Dans les stances d'Antigone, la princesse s'adresse, le plus souvent, à elle-même. Dans la première strophe, qui évoque la mort comme seule solution, elle commence par se désigner par un «tu » qui remplace son «je » de "princesse infortunée» (v. 1331). Elle tente, ainsi, de mettre de la distance entre son «moi» locuteur et son «moi» destinataire. Le premier constate, «Tes Frères sont aux mains » (v. 1336), et s'interroge, « À de nouveaux malheurs te veux-tu réserver?» (v. 1335), alors que le deuxième subit. C'est le «tu» qui «verse [...] des larmes» (v.1339) et qui "devrai[t] se "percer le flanc» (v. 1338). Mais dans la deuxième strophe où elle a, à nouveau, le choix entre la vie et la mort, la princesse décide de regagner son «moi ». Elle s'adresse alors à elle-même à la première personne du singulier. Le choix est bien le sien, et non celui d'un «je » lointain devenu « $\mathrm{tu}$ » : « Dois-je vivre, dois-je mourir ?» (v. 1343).

En passant du «tu » au « je », Antigone réussit à reprendre ses esprits et à assumer la réalité et les choix qui lui sont offerts.

11 Mithridate, quant à lui, débute son premier monologue à la première personne du singulier: «Je ne le croirai point?» (v. 1007). Ce cri du cœur, présenté sous forme de question, montre à quel point le roi est perdu. Dans le deuxième vers, «Tu ne le crois que trop, malheureux Mithridate» (v. 1008), il répond à la question, établissant une sorte de dialogue - question / réponse - entre le «moi» lucide du roi et le «moi » blessé de l'amant qui, le temps d'une réprimande, devient « tu ».

La situation est la même dans son deuxième monologue, où il transforme, encore une fois, son « je » égaré en un «tu» récepteur, pour pouvoir s'asséner la réalité : « Tu vas sacrifier, qui, malheureux ? Ton Fils? » (v. 1394).

Dans les deux vers ci-dessus (1008 et 1394), nous voyons un Mithridate conscient de la situation qui s'adresse à un autre Mithridate, "malheureux ", qui, lui, se voile la face pour ne pas affronter la réalité. L'emploi du terme «malheureux » est, dans ces deux vers, assez expressif, puisqu'il passe du statut d'adjectif à celui de substantif. En qualifiant Mithridate, "malheureux» prend le sens d'«infortuné» ( $4^{e}$ éd. Académie), alors que, employé comme nom, et remplaçant, par là même, "Mithridate ", il étend son champ sémantique : "homme misérable ", le "malheureux» est également un " méchant homme, un homme vil et méprisable " ( $6^{6}$ éd. Académie), voire un "insensé, un fou ». L'opposition entre les deux facettes du personnage est ainsi mise en relief. Après ces deux moments de faiblesse, le roi se ressaisit en reprenant possession de son « je » et en exposant la situation dans toute sa cruauté, puisque dans le reste de ses monologues, il parlera de lui-même avec le « je » de la lucidité : «Quelle faiblesse à moi » (v. 1017), et de la responsabilité : « Je le dois, je le puis » (v. 1389).

Titubant dans son dernier monologue, il ira même jusqu'à se demander, dans un moment de doute profond: "Suis-je Mithridate?»(v.1383), sans plus tenter de se dissimuler derrière un « tu » hypocrite.

Dans le monologue d'Hermione, l'évolution du «moi » de l'émetteur est remarquable. La scène s'ouvre sur une suite d'interrogations à la première personne, "Où suis-je? Qu'ai-je fait?» (v. 1401), et montre ainsi un personnage perdu, cherchant des réponses. Quand Hermione reprend quelque peu ses esprits, elle se donne des ordres à la première personne du pluriel, seule possibilité grammaticale pour s'adresser à soi- 
même à l'impératif : «Non, ne révoquons point l'arrêt de mon courroux. » (v. 1415) : «Il faut [...] qu'elle se conçoive comme dédoublée pour donner et recevoir à la fois l'ordre » (Larthomas, 1980 : 376).

Mais loin de se limiter à cet usage du " nous ", elle se l'attribue entièrement : « il ne vit plus pour nous" (v. 1416). Ici, le pronom de la première personne du pluriel la désigne elle seule. Amplifié par ce passage du singulier au pluriel, l'égo d'Hermione se gonfle ainsi d'orgueil bafoué, donc de colère. Retombant dans le «je » de la solitude pour quelques vers, «Il me laisse » (v. 1425), Hermione réutilise le «nous » dans un impératif qui se veut convaincant : « Non, non, encore un coup, laissons agir Oreste » (1426).

Ce mode verbal, généralement preuve d'autorité et de certitude, devient ici «le signe [...] de l'indécision et d'un désarroi douloureux» (Ratermanis, 1972 : 404). Le summum du délire du personnage est atteint lorsque la princesse évoque «la Mort» (v. 1430) de Pyrrhus, utilisant, après 29 vers de sous-entendus, le terme même. Là, incapable d'assumer une telle responsabilité, refusant même la possibilité d'y être mêlée, elle rejette et le «je » et le "nous », et revêt la troisième personne ou la "non-personne » (Benveniste, 1974 : 255). C'est ainsi qu'elle parle d'elle-même en utilisant son prénom : "Sa mort sera l'effet de l'amour d'Hermione?» (v. 1430).

Elle sort ainsi d'elle-même et devient une autre, une étrangère dont l'amour serait meurtrier. Ce rejet de l'identification pronominale avec soi reflète celui, plus profond, de la culpabilité. En choisissant de devenir une "troisième personne», Hermione se détache de la réalité car "alors que je jure est un engagement, il jure n'est qu'une description » (Benveniste, 1966 : p. 265). Elle préfère donc dire, d'une certaine manière, "elle tue " et ne faire qu'une description détachée de cette action, plutôt que de s'approprier l'acte par un "je tue " accablant et insupportable. D'ailleurs, après être devenue une « Hermione » étrangère, elle n'accepte de réintégrer son «je » que pour évoquer son amour pour Pyrrhus :

Ce Prince, dont mon coeur se faisait autrefois,

Avec tant de plaisir, redire les Exploits (v. 1431 à 1432).

et ne recouvre sa lucidité que pour refuser l'idée de sa mort : «Ah! devant qu'il expire... » (v.1437).

\section{L'apostrophe : Antigone, Jocaste, Roxane, Atalide, Thésée, Phèdre, ...}

Quand leurs propres moyens leur semblaient insuffisants, les anciens faisaient appel à une force supérieure qu'ils croyaient capable de les aider à résoudre leurs problèmes. Souvent, le destinataire de substitution est donc transcendant. Ils utilisent pour cela l'apostrophe définie par Le Petit Robert, comme «figure de rhétorique par laquelle un orateur interpelle tout à coup une personne ou même une chose qu'il personnifie ».

Cette figure est utilisée « lorsqu'un homme étant extraordinairement ému se tourne de tous côtés, [...] s'adresse au Ciel, à la terre, aux rochers, aux choses insensibles » (Lamy, cité par Hawcroft, 1992 : 402), ou « à un autre objet, naturel ou surnaturel, absent ou présent, vivant ou mort, animé ou inanimé, réel ou abstrait, ou [...] à soi-même " (Fontanier, $1977: 371$ ).

Même si l'utilisation de cette figure typique du dialogue semble paradoxale dans le monologue, elle y est récurrente, voire «la plus utilisée » (Larthomas, 1980 :374), et sert 
à "théâtraliser le monologue, en le rendant mouvementé et dynamique» (Hawcroft, 1992 : 409).

Nombreux sont les personnages qui, emportés par la passion de leur monologue, s'adressent au "Ciel», entité supérieure et puissante. Ils espèrent, ainsi, voir leurs vœux exaucés. La parole devient ainsi une prière ascendante, adressée à l'au-delà, et dite tout haut pour que la voix porte :

\section{ANTIGONE :}

Et si tu prends pitié d'une flamme innocente,

ÔCiel! En ramenant Hémon à son Amante,

Ramène-le fidèle, et permets en ce jour. (v. 339 à 341)

JOCASTE :

Ô Ciel! Que tes rigueurs seraient peu redoutables,

Si la foudre d'abord accablait les coupables. (v. 679 à 680).

Sous des apparences de soumission, Antigone essaie d'amadouer le ciel pour qu'il lui soit favorable. Elle lui demande d'avoir « pitié » d'elle, elle qui est « innocente », pour enfin lui asséner deux impératifs, " ramène » et " permets ", exigeants.

Sa mère, à plusieurs scènes d'intervalles, interpelle le même «ciel» en utilisant la même interjection " $\hat{O}$ ». Elle aussi tutoie cette entité supérieure mais ne va pas jusqu'à lui donner des ordres. Jocaste se contente d'exposer une situation imaginaire idéale à travers laquelle elle semble donner des conseils au ciel, en lui proposant d'«accabl[er] [d'abord] les coupables».

Pour Roxane et Atalide, la situation semble plus désespérée. Toutes les deux sont perdues et n'espèrent trouver de réponse qu'en s'adressant au « Ciel » :

ROXANE :

Ô Ciel! À cet affront m'auriez-vous condamnée ? (v. 1070)

ATALIDE :

Ciel, aurais-tu permis que mon funeste amour

Exposât mon Amant tant de fois en un jour. (v. 1435 à 1436).

N'arrivant pas à croire ce qui leur arrive, elles emploient des phrases interrogatives qui expriment leur incrédulité et leur désespoir. Les questions, formulées au conditionnel, sous-entendent des sentiments : «Ô ciel! serait-il possible que vous m'ayez condamnée à cet affront ? ", semble dire Roxane ; "Ciel, serait-il possible que tu aies permis ... », semble lui répondre Atalide.

La seule différence entre les deux rivales est que la première vouvoie le ciel, en un élan d'humilité, alors que la deuxième, accablée de malheurs, le tutoie. Ce «Ciel » que tous sollicitent garde le silence, il « se tait et reste inflexible» (Poulet, IV, 1952: 70), alors que tous l'interpellent par une apostrophe suppliante située, sans exception, en début de vers.

Face au silence céleste, certains préfèrent s'adresser aux «dieux » réunis pour qu'ils exaucent leurs vœux. Jocaste, n'ayant pas reçu de réponse du ciel, tente à nouveau sa chance :

JOCASTE :

Et toutefois, ô Dieux, un crime involontaire, Devait-il attirer toute votre colère? (v.687-688) Toujours aussi déterminée, elle adresse, encore une fois, un reproche à l'au-delà. Débutant sa phrase par l'adverbe d'opposition restrictive, "toutefois ", elle remet en question le jugement divin, en doutant de la justesse de sa décision. L'emploi du verbe «devoir " à la forme interrogative signifie que la violence de la réaction n'était pas justifiée. Jocaste espère que "finalement le ciel sera [...] assez équitable pour décharger 
l'homme, victime innocente, et confesser sa propre culpabilité. [...]. Mais cet espoir est vain " (Poulet, IV, 1952: 70).

Dans Phèdre, tous les monologuistes sollicitent les dieux :

THÉSÉE :

Justes Dieux, qui voyez la douleur qui m'accable,

Ai-je pu mettre au jour un Enfant si coupable? (v. 1165 à 1166)

PHÈDRE :

Ah, Dieux! Lorsqu'à mes voux l'Ingrat inexorable

S'armait d'un ceil si fier, d'un front si redoutable,

Je pensais qu'à l'amour son cour toujours fermé

Fût contre tout mon sexe également armé. (v. 1205 à 1208)

CENONE :

Ah Dieux! Pour la servir j'ai tout fait, tout quitté.

Et j'en reçois ce prix ? Je l'ai bien mérité. (v. 1327 à 1328)

Le roi, respectueux, fait précéder son apostrophe de l'adjectif «justes» qui atteste la « légitim[ité] » de ces Dieux en évoquant leur « équité ». Faisant appel à leur sens de la justice, il se remet en question et leur demande de trancher : peut-il avoir eu un fils si coupable?

Même aveuglé par "la douleur", lui aussi garde un "cœur de père " et endosse l'entière responsabilité des actes d'Hippolyte. En effet, il demande si son «je » a pu " mettre au jour un enfant si coupable", ramenant la culpabilité à sa source, donc à la naissance. Mais, en réponse,

Le silence des dieux lui enlève toute chance d'être reconnu innocent. [...] [En effet,] l'homme

[...] est toujours coupable, soit de son propre chef, soit parce que corrompu, dès avant ses

crimes, par l'acte même, l'acte peut-être mauvais, qui lui donne le jour. (Poulet, IV, 1952 :

70-71).

Ces mêmes dieux, Phèdre n'hésite pas à les interpeller pour qu'ils soient témoins de ses paroles.

L'apostrophe «Ah Dieux!» se situe au début d'une phrase de quatre vers dans laquelle l'héroïne expose ses pensées les plus secrètes, sans plus évoquer l'instance divine. Celle-ci se trouve ainsi en position de témoin passif à qui il n'est rien demandé, sinon de regarder.

En double parfait de Phèdre, CEnone fait également appel au regard des « dieux », selon la même formule «Ah Dieux! ». Ici, témoins de l'« émotion vive [et] profonde [de cette] âme fortement affectée » (Fontanier, 1977 : 372), les divinités observent. Elle ne leur demande rien non plus, et accepte son sort : «je l'ai bien mérité».

Ainsi, c'est à une force transcendante que s'adressent, chacun à sa manière, les personnages isolés le temps d'un monologue. Ils espèrent certes, obtenir ainsi de l'aide, mais ce qu'ils veulent par dessus tout, c'est conjurer la solitude qui est alors la leur.

Par ailleurs, si les monologuistes s'inventent des interlocuteurs, c'est sans doute pour rendre plus acceptable le poids du vide qui les entoure. Mais souvent, ce qu'ils disent, ils aimeraient qu'il soit entendu par la personne qui hante leur esprit. C'est ainsi que leur destinataire prend le visage de cet "Autre absent, mort, disparu» (Ubersfled, III, 1996 : 22) ou interdit, qu'ils n'osent affronter ou qu'ils ne peuvent rencontrer.

Redoutant tous deux le moment de l'aveu, Antiochus et Titus répètent, dans leurs monologues, cette scène tant redoutée. Cette "avant-première " les prépare donc à affronter Bérénice qu'ils imaginent alors devant eux, pour que la " répétition » soit plus réaliste. Titus semble craindre la présence de la reine qu'il maintient loin de lui par le pouvoir de la parole. 
21 démonstratif « ces yeux », comme pour s'obliger à ne pas citer directement la reine : en n'employant pas le possessif « ses ", il lui semble sans doute la maintenir à distance. Il use donc la prosopopée qui consiste à «prendre pour confidents, pour témoins [...] les absents, les morts » (Fontanier, $1977:$ 404).

La situation est la même lorsqu'il évoque le "cœur» de Bérénice accompagné du déterminant indéfini « un coeur » (v.999); l'indéfini brouillant l'image sinon trop nette de la bien-aimée. D'ailleurs, quand il mime ce qu'il va lui dire, "Pourrai-je dire enfin », il n'évoque pas le C.O.I. du verbe « dire " parce que cela aurait équivalu à l'évoquer, elle. La désignant comme « la Reine » (v. 1007), l'article défini assure sa légitimité mais, par là même, son éloignement, Titus ne peut s'empêcher d'en faire, dix vers plus loin, «ta Reine » (v. 1017), donc la sienne puisqu'il s'adresse à lui-même.

Mais cette deuxième personne, quoique jouant le rôle d'un possessif, garde Bérénice à distance puisqu'elle désigne un destinataire que Titus éloigne de sa personne - même si c'est lui-même -, refusant d'employer la première personne qui l'aurait trop impliqué. Ce besoin qu'il a de la présence de la reine se traduit encore plus nettement dans les deux "Bérénice » (v. 988 et 1021) qu'il laisse échapper à la première et à la dernière évocation de sa belle. Ainsi, essayant par tous les moyens verbaux de ne pas rapprocher Bérénice de lui, par peur de souffrir davantage, Titus ne peut pourtant s'en empêcher.

Pour Antiochus, la situation est différente. La reine envahit petit à petit son esprit jusqu'à « apparaître » devant ses yeux. Il débute son monologue en évoquant Bérénice à travers le pronom de la troisième personne : « lui » (v. 20 et 31), « elle » (v. 24, 28 et 35), «la» (v. 27, 29 et 37). Ce pronom est, à proprement parler, celui de la «non-personne ». Bérénice habite les pensées d'Antiochus mais elle est considérée comme absente, lointaine, image même de la prosopopée, et ce n'est qu'au vers 38 qu'elle apparaît réellement :

\section{ANTIOCHUS :}

Belle Reine, et pourquoi vous offenseriez-vous?

Viens-je vous demander que vous quittiez l'Empire;

Que vous m'aimiez? Hélas! Je ne viens que vous dire, (v. 38 à 40)

Antiochus l'interpelle directement: "Belle reine", et s'adresse à elle à la deuxième personne du pluriel, "vous ", utilisant l'apostrophe. En lui attribuant la deuxième personne, considérée comme «partenaire » (Benveniste, 1974:262) du « je », Antiochus fait de Bérénice sa moitié indissociable. En effet, « aucun des deux termes ["je» et «tu»] ne se conçoit sans l'autre; ils sont complémentaires» (Benveniste, 1974: 259). Antiochus semble, d'ailleurs, fasciné par le pronom «vous » qui matérialise sa bien-aimée et la rapproche de lui, puisque dans les trois vers cités, il le répète six fois, sans compter les trois occurrences du morphème porteur de l'indication de personne «ez » dans les désinences verbales de " offenseriez », « quittiez » et " aimiez ».

Dans Alexandre le Grand, Axiane, isolée dans le palais, se retrouve séparée de son bienaimé. Comme elle le croit mort, elle désespère de pouvoir lui reparler un jour. C'est pourquoi elle profite de son monologue pour établir un dialogue fictif avec lui :

\section{AXIANE :}

On m'observe, on me suit. Mais Porus, ne crois pas

Qu'on me puisse empêcher de courir sur tes pas.

Sans doute à nos malheurs ton Cœur n'a pu survivre.(v. 991 à 993) 
S'adressant directement à lui, elle commence par l'interpeller par son prénom, se servant ainsi de l'apostrophe pour le matérialiser devant elle. Elle utilise ensuite la deuxième personne du singulier « $t u$ » pour lui parler, tout au long de son monologue. Récurrentes dans la majorité des 47 vers de la scène, les désignations de Porus ( $37 \mathrm{au}$ total) hantent l'esprit d'Axiane qui, volubile, s'imagine assise face à celui qu'elle aime. Elle se remémore les moments passés avec lui, «lorsque tes yeux, aux miens découvrant ta langueur» (v. 999), et les revit intensément par le pouvoir de la parole qui rend présent le passé.

L'autre à qui l'on parle n'est pas toujours l'amant, mais il est souvent un être aimé. Pour Mithridate (Mithridate, III, 4 ; III, 6 et IV, 5) et Thésée (Phèdre, IV, 3), dont les situations sont similaires, la colère a une même source : tous deux croient que leur bien-aimée les trompe avec leur fils. Les deux personnages, en proie à une même douleur, souffrent autant dans leur cœur de père que d'amant, et leurs monologues sont, sans exception, adressés au sang de leur sang :

\section{MITHRIDATE :}

Pharnace, Amis, Maîtresse? Et toi, mon Fils, aussi ?

Toi de qui la vertu consolant ma disgrâce... (v. 1014-1015)

Ah Fils ingrat! Tu vas me répondre pour tous.

Tu périras. Je sais combien ta Renommée,

Et tes fausses vertus ont séduit mon Armée. (v. 1118 à 1120)

Ô Monime! Ô mon Fils! Inutile courroux! (v. 1413)

THÉSÉE :

Misérable, tu cours à ta perte infaillible.

$[\ldots]$

Un Dieu vengeur te suit, tu ne peux l'éviter.

Je t'aimais. Et je sens que malgré ton offense,

Mes entrailles pour toi se troublent par avance.

Mais à te condamner tu m'as trop engagé. (v. 1157 et v. 1160 à 1163)

On assiste, ici, à la fureur amère de deux pères désespérés. Chacun s'adresse à son fils coupable de l'avoir déçu et trahi dans son amour-propre. Pourtant, l'accusé n'est pas présent et le juge semble répéter la sentence avant de la rendre publique : «tu vas me répondre pour tous» (v.1118), déclare Mithridate, laissant à l'interpellé le droit de se défendre; mais il enchaîne aussitôt, «Tu périras " (v.1119), sans prendre la peine d'écouter le plaidoyer de la défense.

Thésée tranche également, "Un Dieu vengeur te suit, tu ne peux l'éviter» (v.1160), « condamn[ant] » ainsi Hippolyte.

L'omniprésence $d u$ «tu » transforme le monologue en véritable face-à-face entre le père et le fils. Par ce pronom, la furie du roi se déchaîne sur un destinataire absent, donc à l'abri. L'amour paternel est ainsi exprimé indirectement: derrière la sentence acérée, se cache un cœur de père qui «aim[e]» (Mithridate, v. 1391 et Phèdre v. 1161), « se trouble» (Phèdre, v. 1162), et finit par trouver son «courroux [inutile]» (Mithridate, v. 1413). En exorcisant les démons de la vengeance dans la sécurité de la solitude, le père veut, inconsciemment, éviter cette scène à son fils. Il la vit donc pour les deux, jouant tous les rôles, souffrant pour deux, et condamnant l'absent qui, peut-être, ne sera jamais avisé de sa sentence.

Une approche conversationnelle du monologue chez Racine a ainsi permis de mettre en évidence la complexité pragmatique de cette forme dramatique. La parole étant, par définition, destinée à être entendue, le monologuiste, même isolé, s'adresse donc bien à quelqu'un. Chez Racine, les diverses identités possibles de ce destinataire caméléon ont 
pu montrer que son visage prend les traits de l'absent, de la divinité ou, plus simplement, d'un miroir.

\section{BIBLIOGRAPHIE}

BAKHTINE Michaïl, Esthétique de la création verbale, Paris, Gallimard, coll. « Bibliothèque des idées », 1984.

BENVENISTE Emile, Problèmes de linguistique générale T.I, Paris, Gallimard, coll. « Tel », 1974 (1966). DAVID Martine, Le théâtre, Paris, Belin, coll. « Sujets », 1995. FONTANIER Pierre, Les figures du discours, Paris, Flammarion, 1977.

GIRARD G., OUELLET R., RIGAULT C., L'univers du théâtre, Paris, Puf, coll. « Littératures modernes ", 1986.

HAWCROFT Michael, Racine, Rhetoric, and Theatrical Language, Oxford, Oxford University, 1992. HUBERT M.-C., Le théâtre, Paris, Armand Colin, 2003.

JAKOBSON R., Essais de linguistique générale, Paris, Minuit, 1963. LAMY Bernard, La rhétorique, ou, l'Art de parler, Paris, Honoré Champion, 2000.

LARTHOMAS P., Le langage dramatique, Paris, Puf, 1980. MAINGUENEAU D., Pragmatique pour le discours littéraire, Paris, Dunod, 1990.

MAURON C., L'inconscient dans l'œuvre et la vie de Racine, Paris, José Corti, 1969.

MOESCHLER J. et REBOUL A., « Analyses linguistique et littéraire du texte théâtral », Cahiers de linguistique française, 1985, $\mathrm{n}^{\circ}$ 6, Discours théâtral et analyse conversationnelle, Université de Genève, pp. 3-9.

PAVIS P., Le dictionnaire du théâtre, Paris, Éditions Sociales, 1987. PIAGET Jean, Le langage et la pensée chez l'enfant, Paris, Delachaux et Niestlé, coll. « Actualités pédagogiques et psychologiques ", 10 é édition, 1948 (1997).

POULET G., Études sur le temps humain T.I et T.IV, Paris, Librairie Plon, 1952.

RATERMANIS J.B., Essai sur les formes verbales dans les tragédies de Racine, étude stylistique, Paris, A.G. Nizet, 1972.

UBERSFELD Anne, Lire le théâtre I., II et III, Paris, Belin, 1996.

\section{Corpus :}

RACINE Jean, Théâtre-Poésie, Paris, Gallimard, coll. "Bibliothèque de la Pléiade”, édition présentée, établie et annotée par Georges Forestier, 1999. 


\section{NOTES}

1. La même analyse pourrait s'appliquer au deuxième niveau, si celui-ci est considéré également comme celui de la communication entre les acteurs eux-mêmes (sous les traits de leurs rôles respectifs).

\section{RÉSUMÉS}

Dans la majorité des pièces raciniennes, nous retrouvons des scènes dans lesquelles un personnage, seul sur scène, parle ou se parle. Il serait intéressant de voir dans quelle mesure on peut parler seul, sachant que la parole sert, par définition, à communiquer. Ne pouvant sans doute se résoudre à parler pour rien, le personnage se prend à imaginer des destinataires possibles - réels ou imaginaires - mais invariablement absents. Le schéma de la communication est alors dévié de ses caractéristiques initiales et nous assistons à un véritable jeu des pronoms personnels qui s'efforcent de désigner les divers pôles de la communication, se démêlant entre un « je » Ôcaméléon et un « tu » volatile.

In the majority of Racine's plays, we find monologues, scenes where a character stands alone and talks to himself. It would be interesting to see to what extent we can talk alone, knowing that by definition, talking is meant for communicating. The character, probably unable to accept the fact of talking alone, imagines real or imaginary addresses. The communication scheme is thus deviated from its initial characteristics and we notice a real play of personal pronouns designating various communication poles, swinging between a chameleon "I" and a volatile "you".

INDEX

Mots-clés : monologue, dialogue, stylistique, Racine (Jean)

Keywords : monologue, Racine (Jean), stylistics, dialogue

\section{AUTEUR}

\section{CHRISTELLE STEPHAN HAYEK}

Université Saint Esprit de Kaslik - USEK Liban 